\title{
Hold tight or you'll fall off: CD151 helps podocytes stick in high-pressure situations
}

\author{
Ambra Pozzi and Roy Zent \\ Division of Nephrology, Department of Medicine, Vanderbilt Medical Center and VA Medical Center, Nashville, Tennessee, USA.
}

\begin{abstract}
Glomerulosclerosis is a general term for scarring of the kidney glomerulus. It cannot be reversed. As glomerulosclerosis accumulates, the diseased kidney progresses to end-stage renal disease. Treatment with inhibitors of the renin-angiotensin system often decreases the rate of progression of glomerulosclerosis in chronic kidney diseases. Although the mechanisms by which these inhibitors mediate their beneficial effects are incompletely understood, it has been suggested that they act, at least in part, by reducing intraglomerular blood pressure and thereby shear stress-induced loss of podocytes, a key component of the glomerular filtration barrier. In this issue of the JCI, Sachs and colleagues provide experimental confirmation of the critical role of tight adhesion of podocytes to the glomerular basement membrane for maintaining glomerular integrity and provide evidence that inhibition of the renin-angiotensin system reduces glomerulosclerosis in animals with less tightly adherent podocytes, presumably by reducing intraglomerular blood pressure.
\end{abstract}

The kidneys are constantly exposed to $20 \%$ of the circulating blood. This is filtered by individual nephrons, which are composed of the glomerulus (the filtering unit) and the tubules, which reabsorb most of the filtrate. The glomerulus is a unique structure that consists of a capillary bed composed of (a) specialized endothelial cells; (b) mesangial cells, modified mesenchymal cells whose primary function is to maintain the 3-dimensional structure of the capillary bed; (c) highly differentiated visceral epithelial cells called podocytes; and (d) the glomerular basement membrane (GBM), which is found between the podocytes and the endothelial cells (Figure 1). The fenestrated endothelial cells, the podocytes, and the GBM form the glomerular filtration barrier. The podocytes and the GBM are believed to play the most critical roles in maintaining the integrity of the glomerular filtration barrier.

Loss of podocytes, or their detachment from the GBM, represents one of the key pathogenic pathways associated with the development of glomerulosclerosis, a late event common to most renal diseases that cause glomerular injury, including diabetic

Conflict of interest: The authors have declared that no conflict of interest exists.

Citation for this article: J Clin Invest. 2012; 122(1):13-16. doi:10.1172/JCI61858. nephropathy (1). Intraglomerular capillary pressure is a major determinant of podocyte loss and/or detachment; decreasing intraglomerular capillary pressure by vasodilating the efferent glomerular capillaries is the main therapeutic strategy used to decrease the rate of progression of glomerulosclerosis to end-stage renal disease. Inhibiting the renin-angiotensin system, which plays a vital role in regulating blood pressure within the glomerular capillaries, is the principal therapeutic strategy used to achieve this hemodynamic effect in the clinic today. In this issue of the JCI, Sachs and colleagues demonstrate experimentally the importance of the strength of podocyte adhesion to the GBM for resisting the deleterious effects of high intraglomerular capillary pressure (2). Moreover, their data verify experimentally the link between the ability of inhibitors of the renin-angiotensin system to lower intraglomerular capillary pressure and diminish podocyte loss and/or detachment from the GBM and the subsequent decrease in the rate of glomerulosclerosis progression.

\section{Angiotensin-converting enzyme inhibitors decrease podocyte detachment from the GBM}

In order to study the molecular interactions governing the strength of podocyte adhesion to the GBM and their contribution to maintaining the integrity of the glomerular filtration barrier, Sachs and colleagues deleted the gene encoding CD151, a member of the tetraspanin family of proteins that increases the tightness of integrin-dependent adhesion of podocytes to the GBM, in two different mouse strains (2). In their first set of studies, the authors verified that tight podocyte adhesion to the GBM prevents glomerulosclerosis (Figure 2). C57BL/6 mice are usually resistant to glomerular damage, even under conditions of severe hypertension (3); however, Sachs and colleagues found that mice on this background lacking CD151 developed renal injury after induction of severe hypertension. To confirm the link between tight podocyte adhesion to the GBM and prevention of glomerulosclerosis, the authors backcrossed the podocyte-specific CD151-deficient mice onto the renal injury-susceptible FVB background. Unlike the CD151-deficient null mice on the C57BL/6 background, these mice developed spontaneous glomerular injury. When these mice were treated with drugs able to lower systemic and intraglomerular blood pressure (angiotensin-converting enzyme [ACE] inhibitors), they were protected from glomerular disease. In contrast, mice treated with drugs only able to decrease systemic pressure (calcium channel blockers) did not show any protection from glomerular disease. These observations strongly suggest that podocyte adhesion to the GBM promoted by CD 151 is required to prevent the development of glomerular disease under conditions of high intraglomerular capillary pressure and that ACE inhibitors induce their therapeutic effects, at least in part, by decreasing podocyte detachment from the GBM. Although this is a reasonable conclusion based on the known actions of ACE inhibitors, Sachs and colleagues never performed the difficult experiments to directly demonstrate a difference in intraglomerular pressures between the groups of mice treated with different antihypertensives (ACE inhibitors versus calcium channel blockers). This represents a pitfall 


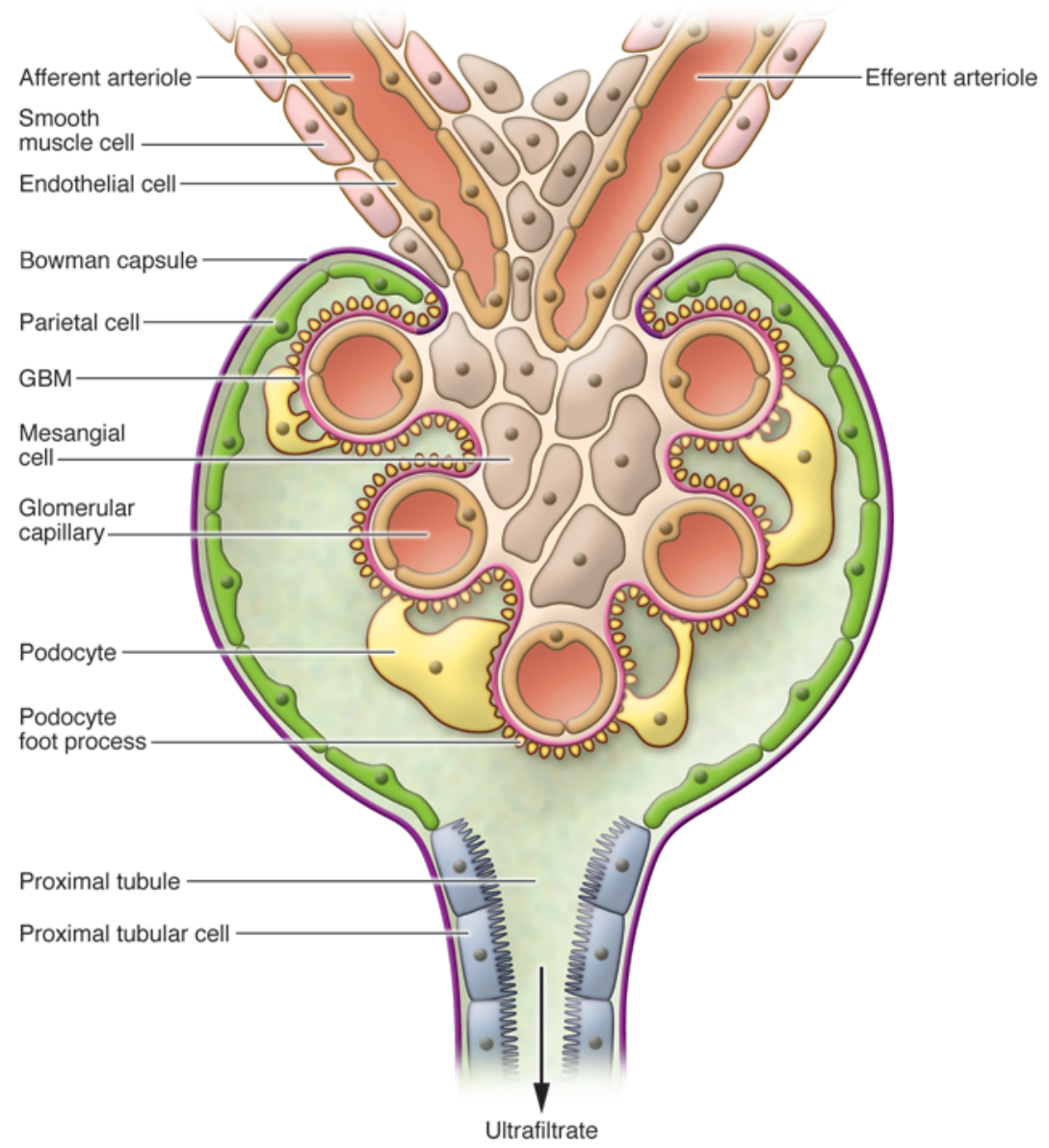

Figure 1

Major structural components of the glomerulus, the filtering unit of the kidney. A key function of the kidneys is to filter the blood, removing waste products and regulating electrolyte concentrations and acid-base balance. The blood is filtered by individual nephrons, composed of the glomerulus (the filtering unit) and the tubules, which reabsorb most of the ultrafiltrate. The glomerulus consists of a capillary bed composed of specialized endothelial cells, mesangial cells, podocytes, and the GBM. The fenestrated endothelial cells, the podocytes, and the GBM form the glomerular filtration barrier.

with the study, as it is possible that ACE inhibitors could also induce their beneficial effects on glomerular disease by acting on glomerular cells directly (1).

\section{$\alpha 3 \beta 1$ is the key integrin mediating podocyte adhesion to the GBM}

The molecular interactions that Sachs and colleagues focused their study on are those mediated by integrins and the tetraspanin CD151. Integrins are a large family of heterodimeric transmembrane proteins, each of which is composed of an $\alpha$ and a $\beta$ subunit. Every integrin binds to a restricted number of ECM ligands (4). The laminin receptor integrin $\alpha 3 \beta 1$ is the most highly expressed integrin on podocytes, although low levels of the collagen receptors integrin $\alpha 1 \beta 1$ and integrin $\alpha 2 \beta 1$ are also present (5). The $\alpha 6$-containing laminin-binding integrins (i.e., $\alpha 6 \beta 1$ and $\alpha 6 \beta 4)$ are not expressed in mature podocytes (5). Integrin-dependent binding has been shown to be critical for podocytes to adhere to the GBM, and mice lacking the integrin $\beta 1$ subunit in podocytes die at approximately 5-6 weeks of age as a result of progressive podocyte loss $(6,7)$. A similar but less severe phenotype is observed when the gene encoding integrin $\alpha 3$ subunit is deleted in podocytes (8). Mice with podocyte-specific deficiency in either $\alpha 3$ or $\beta 1$ develop massive proteinuria caused by focal glomerulosclerosis and disorganization of the GBM (6-8). These data strongly suggest that integrin $\alpha 3 \beta 1$ is the $\beta 1$ integrin key to mediating the binding of podocytes to the GBM.

\section{CD151 increases the tightness of integrin $\alpha 3 \beta 1$-dependent adhesion to the GBM}

It has been clearly demonstrated that podocyte adhesion to the GBM via integrin $\alpha 3 \beta 1$ is enhanced by a mechanism that requires interaction of the integrin with the tetraspanin protein CD151 (8). CD151 interacts with all the laminin-binding integrins and regulates the tightness of integrin-dependent adhesion, cell morphology, and cell migration (9). This interaction has clinical significance because patients homozygous for a nonsense mutation in the gene encoding CD151 develop glomerulosclerosis, sensorineural hearing loss, epidermolysis bullosa, and thalassemia (10). Interestingly, global deletion of Cd151 in mice induces focal segmental glomerulosclerosis that resembles that observed in humans with nonsense mutations in CD151; however, these mice do not develop skin impairment or hearing loss (8). Furthermore, the phenotype of glomerulosclerosis is almost identical to that observed in mice lacking the integrin $\alpha 3$ subunit in podocytes (8).

Sachs and colleagues now provide a molecular explanation for the clinical observation that humans with nonsense mutations in CD151 develop glomerulosclerosis by demonstrating that expression of CD151 in human podocytes is localized to the podocyte-GBM interface (2). This suggests that, as in mice, CD151 regulates podocyte adhesion to the GBM and that the nonsense mutations have catastrophic consequences as a result of decreased podocyte adhesion to the GBM. In additional mouse studies, the authors investigated the molecular interactions underlying this and show that the podocyte loss and glomerulosclerosis that arise as a result of specifically deleting Cd151 in podocytes is only dependent on its interactions with integrin $\alpha 3 \beta 1$. They clearly showed that the tight adhesion of mouse podocytes to the GBM requires a CD151 interaction with integrin $\alpha 3 \beta 1$, is specific for podocyte binding to laminins in the GBM, and is not dependent on increased integrin expression and/or activation. Consistent with these data, they found that CD151 interacts with the integrin $\alpha 3$ subunit at the podocyte-GBM interface in the healthy human kidney. Furthermore, in a set of elegant in vitro and in vivo studies, they showed that CD151 shifts the localization of $\alpha 3 \beta 1$ integrins from focal adhesions into teraspanin 


\section{A WT podocyte}

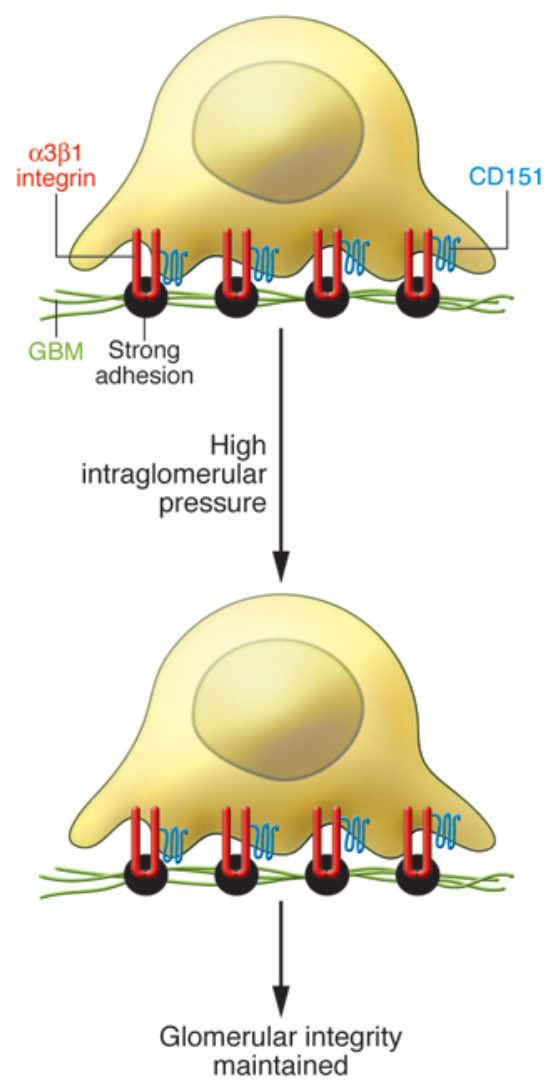

B CD151-deficient podocyte

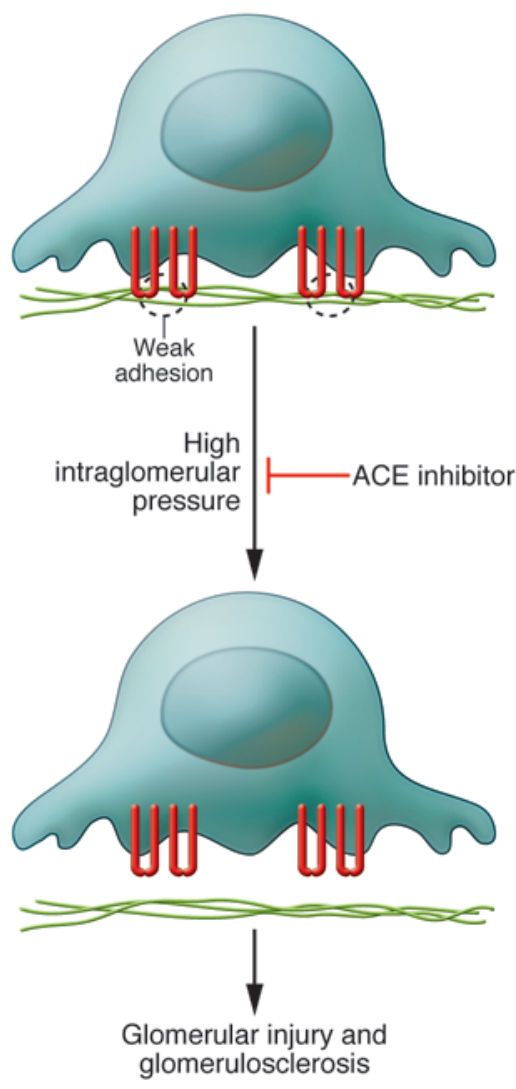

\section{Figure 2}

CD151-deficient podocytes adhere less tightly to the GBM than do wild-type podocytes. (A) Integrin $\alpha 3 \beta 1 / C D 151$ complexes found in diffuse focal adhesions promote strong adhesion of wild-type podocytes to the GBM, resulting in maintenance of the glomerular filtration barrier in high-pressure situations. (B) Deleting the gene encoding CD151 results in large focal adhesions that do not promote tight adhesion of podocytes to the GBM, with consequent damage to the glomerular filtration barrier and glomerulosclerosis in high-pressure situations. ACE inhibitors lower intraglomerular capillary pressure, reducing podocyte loss and/or detachment from the GBM and glomerulosclerosis progression rate. webs at the podocyte-GBM interface. The mechanisms by which CD151 achieves this action are unknown and were not addressed in the study. This is not surprising, as little is known about how the tetraspanins, including CD151, function.

\section{CD151 regulates integrin $\alpha 3 \beta 1$ localization on podocytes}

Tetraspanins are a large family of fourtransmembrane domain proteins (there are 33 tetraspanins identified in humans). They are thought to primarily facilitate the assembly of specialized molecular aggregates on plasma and intracellular membranes, called tetraspanin-enriched microdomains (TEMs), in which numerous protein-protein interactions occur. In the case of CD151, the TEM contains the laminin receptors, phosphoinositide 4-kinase (PI4K), PKC, and other tetraspanins (e.g., CD9, CD81, and CD63) (11). CD151 has been proposed to control different integrin-dependent functions by regulating the trafficking/endocytosis of integrin complexes. This might be mediated by a potential tyrosine-based sorting motif in the $\mathrm{C}$-terminal domain of CD151, which is required for plasma membrane endocytosis and sorts proteins from the trans-Golgi network to lysosomes for degradation (11). Another potential mechanism by which CD151 alters integrin-dependent functions is by its ability to alter Ras activation through a yet-to-bedetermined mechanism (12).

Despite our lack of understanding of the molecular mechanisms by which CD151 regulates integrin localization, it is clear from the work of Sachs and colleagues that when more integrin $\alpha 3 \beta 1$ is localized to focal adhesions, as they saw in CD151-deficient podocytes, the less tightly podocytes can bind to their laminin substrates. The glomerulosclerosis seen in mice lacking CD151 in podocytes is likely caused by podocyte loss; however, it is unclear from the authors' work whether cell detachment is caused by increased podocyte apoptosis or by actual detachment of live podocytes. However, Sachs and colleagues provide good evidence of decreased podocyte numbers in the podocyte-specific CD151-deficient mice. This is consistent with the proposal that massive podocyte detachment from the GBM underlies the similar glomerulosclerosis phenotype seen in mice lacking the integrin $\beta 1$ subunit in podocytes $(6,7)$.

\section{Integrin-dependent tight binding of podocytes to the GBM regulates glomerular disease}

In conclusion, the data reported by Sachs and colleagues have significant implications for both podocyte and integrin biology. They indicate that the strength of the adhesive force anchoring podocytes to the GBM plays a critical role in maintaining the integrity of the glomerular filtration barrier. They also show that in the absence of integrin $\alpha 6 \beta 4$, which is not expressed by podocytes, integrin $\alpha 3 \beta 1$ can mediate tight binding of epithelial cells to a basement membrane so that cells can tolerate high-shear stress environments. Thus, integrins $\alpha 3 \beta 1$ and $\alpha 6 \beta 4$ have distinct roles in mediating tight binding of epithelial cells to basement membranes in the skin and glomerulus, respectively. The effect of abnormal integrin-dependent tight binding of epithelial cells in skin has been well described with respect to the bullous skin diseases (13). The work 
of Sachs and colleagues provides fresh insight into how integrin-dependent tight binding of podocytes to the GBM regulates glomerular disease.

\section{Acknowledgments}

A. Pozzi and R. Zent are supported by an O'Brien Center grant, by NIH grant DK065123, and by merit awards from the Department of Veterans Affairs. R. Zent is funded by NIH grants DK075594, DK65123, and DK083187 and by an American Heart Association established investigator award.

Address correspondence to: Roy Zent or Ambra Pozzi, Room C3210, Medical Center North, Vanderbilt University Medical Center, Nashville, Tennessee 37232-2372, USA. Phone: 615.322.4632; Fax: 615.322.4689;
E-mail: roy.zent@vanderbilt.edu (R.Zent); ambra.pozzi@vanderbilt.edu (A. Pozzi).

1. Campbell KN, Raij L, Mundel P. Role of angiotensin II in the development of nephropathy and podocytopathy of diabetes. Curr Diabetes Rev. 2011;7(1):3-7.

2 . Sachs $\mathrm{N}$, et al. Blood pressure influences end-stage renal disease of Cd151 knockout mice. J Clin Invest. 2012;122(1):348-358.

3. Baleato RM, Guthrie PL, Gubler MC, Ashman LK, Roselli S. Deletion of CD151 results in a straindependent glomerular disease due to severe alterations of the glomerular basement membrane. Am J Pathol. 2008;173(4):927-937

4. Pozzi A, Zent R. Extracellular matrix receptors in branched organs. Curr Opin Cell Biol. 2011;23(5):547-553.

5. Mathew S, Chen X, Pozzi A, Zent R. Integrins in renal development [published online ahead of print May 21, 2011]. Pediatr Nephrol. doi:10.1007/ s00467-011-1890-1.

6. Pozzi A, et al. Beta1 integrin expression by podocytes is required to maintain glomerular structural integrity. Dev Biol. 2008;316(2):288-301.
7. Kanasaki K, et al. Integrin beta1-mediated matrix assembly and signaling are critical for the normal development and function of the kidney glomerulus. Dev Biol. 2008;313(2):584-593.

8 . Sachs N, et al. Kidney failure in mice lacking the tetraspanin CD151. J Cell Biol. 2006;175(1):33-39.

9. Margadant C, Frijns E, Wilhelmsen K, Sonnenberg A. Regulation of hemidesmosome disassembly by growth factor receptors. Curr Opin Cell Biol. 2008;20(5):589-596.

10. Karamatic Crew V, et al. CD151, the first member of the tetraspanin (TM4) superfamily detected on erythrocytes, is essential for the correct assembly of human basement membranes in kidney and skin. Blood. 2004;104(8):2217-2223.

11. Wang HX, Li Q, Sharma C, Knoblich K, Hemler ME. Tetraspanin protein contributions to cancer. Biochem Soc Trans. 2011;39(2):547-552

12. Sawada S, Yoshimoto M, Odintsova E, Hotchin NA, Berditchevski F. The tetraspanin CD151 functions as a negative regulator in the adhesiondependent activation of Ras. J Biol Chem. 2003; 278(29):26323-26326.

13. Margadant C, Charafeddine RA, Sonnenberg A. Unique and redundant functions of integrins in the epidermis. FASEB J. 2010;24(11):4133-4152.

\title{
The flip-flop HuR: part of the problem or the solution in fighting cancer?
}

\author{
Jacqueline C. Shultz ${ }^{1}$ and Charles E. Chalfant ${ }^{1,2,3}$
}

\begin{abstract}
1Department of Biochemistry and Molecular Biology, Virginia Commonwealth University, Richmond, Virginia, USA ${ }^{2}$ Research and Development, Hunter Holmes McGuire Veterans Administration Medical Center, Richmond, Virginia, USA. ${ }^{3}$ The Massey Cancer Center, Virginia Commonwealth University, Richmond, Virginia, USA.
\end{abstract}

\begin{abstract}
Chronic inflammation has long been appreciated to play a critical role in tumor development and maintenance. Among the mechanisms involved in coordinating the initiation and resolution of inflammation are those responsible for modifying mRNA stability and/or translation. Several studies have linked the RNA-binding protein HuR, which increases mRNA stability, with malignant transformation. However, in this issue of the JCI, Yiakouvaki et al. compellingly demonstrate in mice that increased HuR activity in myeloid cells has a protective role in the onset of pathologic intestinal inflammation (i.e., colitis) and colitis-associated cancer (CAC). These observations highlight the need to understand the roles of $\mathrm{HuR}$ in distinct cell populations in vivo and suggest that enhancing $\mathrm{HuR}$ activity may be of clinical benefit in protecting against pathologic inflammation and cancer.
\end{abstract}

It has been clear for some time that chronic inflammation can promote and/or exacerbate tumor initiation, tumor promotion, and tumor progression (1). For example, clinical and epidemiologic studies have indicated that inflammation of the liver as a result of alcohol abuse or chronic infection with hepatitis $C$ virus is associated with hepatocellular carcinoma; inflam-

Conflict of interest: The authors have declared that no conflict of interest exists.

Citation for this article: J Clin Invest. 2012;

122(1):16-19. doi:10.1172/JCI61677. mation of the lung as a result of cigarette smoking, asbestos exposure, or silica exposure is associated with lung carcinoma; and inflammatory bowel diseases (IBDs) such as ulcerative colitis (UC) are associated with colon cancer. Defining the mechanisms responsible for coordinating the initiation and resolution of inflammation is therefore critical to understanding how inflammation-associated cancers arise and progress and to identifying potential therapeutic targets. Among the mechanisms responsible for coordinating the initiation and resolution of inflammation are those controlling the production and post-transcriptional regulation of mRNAs encoding inflammatory mediators. In this issue of the JCI (2), Yiakouvaki and colleagues provide a comprehensive study aimed at clarifying the role of one RNA-binding protein that controls mRNA stability and/or translation (HuR) in inflammation and inflammation-associated cancer.

\section{Post-transcriptional regulation of gene expression by HuR}

HuR is a member of the embryonic lethal, abnormal vision (ELAV) family of RNAbinding proteins that is encoded by the ELAV1 gene. It regulates gene expression via post-transcriptional mechanisms (3-5). Traditionally, HuR functions to modulate the stability and translation of target mRNAs that posses AU-rich elements (AREs) in their $3^{\prime}$-untranslated regions (UTRs). It has been previously demonstrated that myeloid HuR overexpression could induce translational silencing of AREcontaining transcripts, such as Tnfa, even though the mRNAs were stabilized (6). HuR is predominantly nuclear, but in response 